\title{
Entwicklung eines generischen Bewertungsmodells für Geschäftsmodelle der Energiewirtschaft
}

\author{
Nina Germanus ${ }^{1}$ Patrick Granzow' ${ }^{1}$ Benjamin Grosse ${ }^{1}$ · Johannes Kochems ${ }^{1}$ · Johannes Giehl' ${ }^{1}$ (D) \\ Joachim Müller-Kirchenbauer ${ }^{1}$
}

Online publiziert: 19. August 2020

๑) Springer Fachmedien Wiesbaden GmbH, ein Teil von Springer Nature 2020

\section{Zusammenfassung}

Durch Umbrüche im Rahmen der Energiewende und der begleitenden Digitalisierung ergeben sich Chancen für neue Geschäftsmodelle im Energiesystem. Anknüpfend an diese Ausgangslage wird in diesem Artikel eine Methode vorgestellt, mithilfe derer potenzielle Geschäftsmodelle auf ihre Erfolgsaussichten aus Perspektive der betriebswirtschaftlichen Forschung überprüft werden können. Der methodische Ansatz kombiniert im Rahmen eines interaktiven Tools eine qualitative Einschätzung mit einer quantitativen Bewertung unter Anwendung einer Discounted-Cashflow-Methode. Die Methode wird für verschiedene Fallstudien exemplarisch angewendet und ihre Generalisierbarkeit demonstriert. Das Tool lässt es zu, nahezu beliebig konfigurierte Geschäftsmodelle des Energiesektors zu untersuchen.

Schlüsselwörter Geschäftsmodelle · Energiewende · Qualitative Bewertung · Quantitative Bewertung · Virtuelles Kraftwerk $\cdot$ Community Energy $\cdot$ Discounted Cashflow

\section{A Generic Evaluation Tool for Business Models in the Energy Industry}

\begin{abstract}
The energy transformation and the accompanied digitalization create opportunities for new business models in the energy system. This article presents a method that allows to examine the possible business models regarding their potential success in the energy market from a business perspective. In an interactive tool, the methodological approach combines a qualitative assessment with a quantitative evaluation using a discounted cashflow method. The method is applied to several case studies and its generalizability is demonstrated. The tool allows to investigate various business models of the energy sector.
\end{abstract}

\section{Einleitung}

Die Energiewende führt in Deutschland und weltweit zu einer deutlichen Veränderung der bestehenden Wertschöpfungsketten. Neue $\mathrm{CO}_{2}$-neutrale Energieerzeugungstechnologien, flexible Verbrauchseinheiten, Smart Homes, Smart Grids und viele weitere Entwicklungen erfordern eine Umgestaltung traditioneller Geschäftsbeziehungen. Hierbei entwickeln Unternehmen neue Geschäftsmodelle und können in Wertschöpfungsnetzwerken verschiedene Funktionen und Schnittstellen im Energiesystem der Zukunft

Johannes Giehl

giehl@er.tu-berlin.de

1 Fachgebiet Energie- und Ressourcenmanagement, Technische Universität Berlin, Berlin, Deutschland übernehmen. Bei der Breite der Veränderungen sind dabei zahlreiche neue Geschäftsmodelle vorstellbar, wobei ihr Auftreten sowohl förderlich als auch hinderlich für die Zielerreichung eines $\mathrm{CO}_{2}$-neutralen Energiesystems sein kann. Zudem stellt sich die Frage, ob energiewendeförderliche Geschäftsmodelle auch aus einzelwirtschaftlicher Sicht wirtschaftlich sind.

Der vorliegende Forschungsbeitrag präsentiert einen generischen Bewertungsansatz für neue, energiewendeförderliche Geschäftsmodelle aus betriebswirtschaftlicher Sicht. Der Fokus liegt dabei auf der Entwicklung einer generischen Vorgehensweise sowie eines Bewertungsmodells, das auf die verschiedenen Schnittstellen und Geschäftsbeziehungen neuer Geschäftsmodelle adaptierbar ist. Die Lücke zwischen klassischen, dedizierten Geschäftsmodellanalysen in traditionellen Wertschöpfungsketten und dem Bedarf zur schnellen Abschätzung von neuen Geschäftsmo- 
dellen in Wertschöpfungsnetzwerken wird durch die Kombination eines qualitativen und eines quantitativen Ansatzes unter Fokussierung auf sektorenintegrierte und plattformbasierte Geschäftsmodelle geschlossen. Bei der Bewertung wird die Perspektive der betriebswirtschaftlichen Forschung eingenommen. Einzelne Aspekte beim qualitativen Bewertungsteil weiten den Fokus auf, sodass an diesen Stellen volkswirtschaftliche Marktrahmenbedingungen einbezogen werden. Dies ist so angelegt, dass die Auswirkungen auf das Geschäftsmodell bzw. den dahinterstehenden betrieblichen Akteur deutlich werden. Insofern bleibt auch an diesen Stellen der betriebswirtschaftliche Analysefokus gewahrt. Es wird also die Sicht des Unternehmens eingenommen, das das jeweilige Geschäftsmodell realisiert bzw. erwägt, dies zu tun.

Die Analyse zeigt zunächst den Stand der Forschung auf, wobei der Fokus auf Geschäftsmodellen der Energiewirtschaft liegt und stellt im Folgenden das neu entwickelte Evaluationssystem für Geschäftsmodelle (ESGM) vor. Daraufhin wird die Anwendung des Modells beispielhaft dargestellt und abschließend auf die Limitationen sowie weiteren Forschungsbedarf verwiesen.

\section{Status Quo}

Im folgenden Abschnitt werden die Charakteristika von energiewirtschaftlichen Geschäftsmodellen kurz vorgestellt. Zudem wird auf den aktuellen wissenschaftlichen Stand zur Bewertung von Geschäftsmodellen eingegangen. Aus der Betrachtung der beiden Aspekte wird anschließend die Forschungsfrage abgeleitet.

\subsection{Spezifika der Geschäftsmodelle der Energiewirtschaft}

Die aktuellen Geschäftsmodelle der Energiewirtschaft werden durch die Trends und das Zusammenwirken von Defossilisierung, Dezentralisierung und Digitalisierung beeinflusst. Zahlreiche Studien greifen diese Aspekte bereits auf und sortieren sie in die klassische energiewirtschaftliche Wertschöpfungskette und gängige Geschäftsmodellbeschreibungen ein. $\mathrm{Zu}$ nennen sind beispielsweise die Autoren Burger und Luke (2017) oder Löbbe und Hackbarth (2017) zur Entwicklung von generischen Geschäftsmodellen auf Basis des Business Model Canvas von Osterwalder und Pigneur (2013). Eine quantitative Betrachtung der Zahlungsströme und damit finanzielle Bewertung von Geschäftsmodellen als Basis für erfolgsrelevante unternehmerische Entscheidungen ist jedoch nicht durchgeführt worden.

Ein weiterer und im Rahmen einer Vollaufnahme entstandener Ansatz besteht in der Verwendung des Business
Model Frameworks for the Energy Transformation (BMFE). Mittels diesem haben die Autoren Giehl et al. (2020) prototypische Geschäftsmodelle des gesamten energiewirtschaftlichen Wertschöpfungsnetzwerks ermittelt und beschrieben. Das BMFE ist zum Zweck der qualitativen Beschreibung von energiewirtschaftlichen Geschäftsmodellen entwickelt worden. Die Formulierung allgemeiner und energiewirtschaftsbezogener Geschäftsmodellkomponenten ermöglicht eine Beschreibung von Geschäftsmodellen der Energiewirtschaft.

Im Rahmen der Studie sind 17 Geschäftsmodellklassen der energiewirtschaftlichen Wertschöpfung ermittelt worden. Diese enthalten 69 Geschäftsmodellprototypen der Energiewirtschaft und stellen den aktuellen Stand energiewirtschaftlicher Geschäftsmodelle, Geschäftsmodellprototypen und Geschäftsmodellklassen in Deutschland dar. Zudem wird ersichtlich, dass die zunehmende Dezentralität in allen Sektoren zusammen mit digitalen Lösungen den Koordinationsbedarf erhöht. Dies führt zu einer steigenden Komplexität der Wertschöpfung, die zunehmend im Rahmen von Wertschöpfungsnetzwerken unter Anwendung neuer Geschäftsmodelle realisiert wird. So treten beispielsweise neue Unternehmen mit Software-, Automatisierungs- und Plattformlösungen sowie Lösungen der Sektorenkopplung in den Markt ein. Zusätzlich zu der qualitativen Geschäftsmodellbeschreibung mittels des BMFEs ist jedoch durch die Autoren bislang keine quantitative Bewertung der Geschäftsmodelle erfolgt.

\subsection{Geschäftsmodellbewertung}

Die qualitative Beschreibung und damit verbunden eine erste Bewertung kann mittels der zuvor genannten Frameworks erfolgen. Für eine erste Bewertung mit Hilfe solcher Frameworks können die Elemente bereits bei der Einteilung in die verschiedenen Segmente auf einer Skala mit drei Ebenen bewertet werden. Dabei kann ein Element eines Geschäftsmodells mit ,verfügbar/realistisch“, „unsicher/ auszuarbeiten“, oder ,nicht verfügbar/potentielles K.O.Kriterium“"klassifiziert werden (Knab et al. 2014).

Darauf aufbauend lassen sich in der Literatur weitere qualitative Bewertungsmethoden finden. Um Geschäftsmodelle einer ersten Priorisierung zu unterziehen, kann eine zweidimensionale Kosten-Nutzen-Matrix genutzt werden, welche die geschätzte Attraktivität dem geschätzten Aufwand gegenüberstellt. Die folgenden Kriterien können auf einer dreigliedrigen Bewertungsskala in ,hoch“, „mittel“ und ,gering“ eingeteilt werden (Knab et al. 2014).

Für eine Makro-Umwelt-Analyse von Geschäftsmodellen, kann die so genannte PESTEL-Methode verwendet werden. Diese Methode besteht aus sechs Dimensionen, welche in der Literatur als allgemeingültig angesehen werden. Die PESTEL-Methode besteht aus politischen, 
wirtschaftlichen, soziokulturellen, technologischen, Umwelt- und rechtlichen Faktoren. Mit Hilfe dieser Faktoren, kann ein Geschäftsmodell analysiert und bewertet werden (Schallmo 2014a).

Für eine Mikro-Umwelt-Analyse von Geschäftsmodellen, kann die Branchenstrukturanalyse auf Grundlage einer Analyse mittels Porter's five forces herangezogen werden. Mit Hilfe dieser Methode, können die Bedrohungen durch potenzielle Neueintritte, durch Rivalität unter Wettbewerbern, durch Substitutionsprodukte und -dienstleistungen, sowie die Verhandlungsmacht von Abnehmern und Lieferanten analysiert und bewertet werden (Schallmo 2014a; Porter 2008).

Für eine weitere Bewertung von Geschäftsmodellen, lässt sich eine klassische Scoring-Tabelle nutzen. In dieser Scoring-Tabelle werden verschiedene Bewertungskriterien des Geschäftsmodells, wie zum Beispiel der Bedarf an Mitarbeitern, Kapital, Know-How für die Umsetzung, die Lebensdauer, das Umsatzvolumen, oder die Differenzierung gegenüber Wettbewerbern, auf einer fünfstufigen Skala bewertet. Die Gewichtung der Bewertungen kann individuell angepasst werden, um daraus anschließend einen Gesamt-Score zu errechnen (Schallmo 2014b).

Auf der quantitativen Seite besteht eine Möglichkeit, Geschäftsmodelle zu bewerten darin, ihre zukünftigen Zahlungsrückflüsse zu vergleichen. Somit kann bei der Bewertung von Geschäftsmodellen auf die Discounted CashFlow-Methode (DCF-Methode) zurückgegriffen werden. In der energiewirtschaftlichen Grundlagenliteratur wird diese allgemeine Methode aufgegriffen und um spezifische Aspekte der Energiewirtschaft ergänzt. Dazu zählen konkrete Vorgaben der technologiespezifischen Parameter, die Quantifizierung von Kosten und der Umgang mit unsicheren Energiepreisen (Khatib 2014; Konstantin 2009; Quaschning 2015).

Gegenüber der umfassenden Betrachtung von klassischen Investitionen existiert nur eine geringere Anzahl an Arbeiten zur quantitativen Bewertung von Geschäftsmodellen. Im Folgenden werden beispielhaft Arbeiten zu neuen Technologien aus dem Bereich der Digitalisierung und der Sektorenkopplung aufgeführt. Für einzelne plattformbasierte Geschäftsmodelle existieren in der Literatur Wirtschaftlichkeitsbetrachtungen. Böttger et al. (2013) und Loßner et al. (2015) beschreiben modell- und szenariogestützte Ansätze, um die Erlöse von virtuellen Kraftwerken an den Spot- und Regelleistungsmärkten zu prognostizieren. Reif und Fox (2014) analysieren die Wirtschaftlichkeit eines beispielhaft konzipierten virtuellen Kraftwerks für verschiedene Szenarien. Für Cloud-Speicher-Geschäftsmodelle haben Liu et al. (2017) ein ökonomisches Bewertungsmodell entwickelt, welches auf historischen Strompreisen und typischen Kunden-Lastprofilen basiert.
Auch im Fall von Power-to-X-Anwendungen gibt es erste Bewertungsansätze. Diese beziehen sich jedoch auf spezifische Anwendungsfälle. Beispiele sind eine Kostenbewertung der Power-to-Hydrogen-Technologie im Hinblick auf die Salzkavernenspeicherung von Noack et al. (2015) und die wirtschaftliche Bewertung der Power-to-Hydrogenund Power-to-Methan-Technologie in Verbindung mit Systemdienstleistungen von Michaelis et al. (2013).

Zudem nehmen viele Analysen im Gegensatz zur vorliegenden betriebswirtschaftlichen Analyse eine volkswirtschaftliche Sicht auf neue Technologien ein. So bewerten Jentsch und Trost (2014) den optimalen Einsatz der Powerto-Methan-Technologie im Hinblick auf ein $85 \%$-erneuerbare-Energien-Szenario in Deutschland. Auch die Studie von Agora Verkehrswende et al. (2018) zur wirtschaftlichen Bewertung von synthetischen Kraft- und Brennstoffen im Verkehrs- und Wärmesektor besitzt eine Sicht auf das Gesamtsystem und bewertet somit kein Geschäftsmodell.

Ein Blick auf andere Branchen zeigt ein differenzierteres Bild. Die Wirtschaftsinformatiker Anke und Krenge (2016) stellen ein webbasiertes Bewertungstool für datengetriebene Geschäftsmodelle vor. Die Wirtschaftlichkeit einer Idee wird dabei bereits in frühen Planungsphasen entsprechend berücksichtigt. Margaria-Steffen et al. (2015) haben ein ITTool zur Visualisierung und Gestaltung dienstleistungsorientierter Geschäftsmodelle in der Medizin entwickelt. Weiterhin existieren kommerzielle Anwendungen, mit denen Geschäftsmodelle gestaltet und grob auf ihre Wirtschaftlichkeit hin untersucht werden können (DataPartner 2019; PWC 2018).

Zusammengefasst kann festgestellt werden, dass in der energiewirtschaftlichen Forschung viele Ansätze zur Bewertung von Investitionsentscheidungen existieren. Es bestehen jedoch nur wenige generische Ansätze zur Bewertung von neuen oder bestehende Geschäftsmodellen. Die Bewertung von Geschäftsmodellen ist zudem oft an vorher festgelegten spezifischen Fragestellungen für konkrete Anwendungsfälle ausgerichtet und nicht auf andere Anwendungsfälle übertragbar.

\subsection{Forschungslücke}

Aus den vorherigen beiden Abschnitten geht hervor, dass die Geschäftsmodelle der Energiewirtschaft in Deutschland umfangreich beschrieben wurden, jedoch bisher keine weitergehende quantitative Bewertung vorgenommen wurde. Die aktuellen Ansätze der Geschäftsmodellbewertung sind zudem auf einzelne Anwendungsfälle ausgerichtet.

Es besteht somit die Problematik, ausgehend von einer qualitativen Beschreibung eines Geschäftsmodells dieses weitergehend mit einem generischen Ansatz bewerten zu können. Dies betrifft zudem neuartige Geschäftsmodelle der Energiewirtschaft, da dort vor allem qualitative Bewer- 
tungen vorliegen (Dena 2019; BDEW 2017; Zeller 2014). Weiterhin sind die wenigen bestehenden Ansätze zur Wirtschaftlichkeitsbewertung von Geschäftsmodellen auf spezifische Anwendungsfälle zugeschnitten, auf einzelne Technologien angepasst oder berücksichtigen schwerpunktmäBig die Sicht eines bestimmten Unternehmens.

Damit lässt sich die zentrale Fragestellung dieses Papiers wie folgt zusammenfassen:

Wie lassen sich energiewirtschaftliche Geschäftsmodelle auf einer qualitativen Ebene sowie einer quantitativen Ebene mittels eines integrierten, generischen Ansatzes aus betriebswirtschaftlicher Perspektive bewerten?

Die Forschungsfrage umfasst zwei Aspekte. Zunächst geht es um eine qualitative Bewertung von Geschäftsmodellen. Dieser Schritt dient dazu, aus einer Liste von Geschäftsmodellen erfolgsversprechende Geschäftsmodelle auszuwählen. Anschließend gilt es, darauf aufbauend eine generische Methode zur Bewertung der Zahlungsströme und damit eine Quantifizierung der Wirtschaftlichkeit eines Geschäftsmodells aufzustellen.

\section{Generisches Bewertungsmodell für Geschäftsmodelle der Energiewirtschaft}

Die qualitative und quantitative Bewertung von Geschäftsmodellen der Energiewirtschaft erfolgt mithilfe eines toolgestützten Ansatzes. Der Grundansatz ist durch die Einbettung in das Tool so gewählt, dass generisch eine Vielzahl unterschiedlich gearteter Geschäftsmodelle betrachtet werden kann. Das ESGM ist insgesamt zweistufig aufgebaut. Zunächst erfolgt eine Bewertung qualitativer Parameter für (verschiedene) Geschäftsmodelle. Hierdurch können Geschäftsmodelle miteinander verglichen werden. Die prinzipielle Attraktivität von Geschäftsmodellen wird anhand eines generischen Nutzen-Aufwands-Vergleichs ermittelt. Im Anschluss erfolgt die quantitative Bewertung (ausgewählter) Geschäftsmodelle. Hierzu sind detailliertere Informationen hinsichtlich der Geschäftsmodelle (zugrundeliegende Technologien, Produkte und Kosten) zu erfassen und gegenüberzustellen. Für den praktischen Gebrauch empfiehlt sich unter arbeitsökonomischen Gesichtspunkten daher als Ergebnis der qualitativen Analyse eine klare Eingrenzung auf besonders attraktive Geschäftsmodelle, welche in der quantitativen Analyse vertieft analysiert werden. Ziele der Bewertung sind somit einerseits die grundlegende Attraktivität eines Geschäftsmodells aus Unternehmensperspektive zu bestimmen und andererseits mit dem Geschäftsmodell zusammenhängende betriebswirtschaftliche Erfolgskennziffern abzuschätzen. Somit wird für das Un- ternehmen, das das Geschäftsmodell realisiert oder erwägt dies zu tun, eine Entscheidungsvorbereitung getroffen, um eine Abwägung für oder gegen die Ausweitung oder Realisierung eines Geschäftsmodells auf dieser Basis zu treffen. Als attraktiv wird in der qualitativen Bewertung ein Geschäftsmodell angesehen, das aus betriebswirtschaftlicher Perspektive eine hohe und langfristige Rentabilität erwarten lässt und bei dem somit der Nutzen in der NutzenAufwand-Bilanz deutlich überwiegt. Im Folgenden ist das Vorgehen der beiden Analysestufen dargelegt.

\subsection{Qualitative Bewertung von Geschäftsmodellen}

Der erste Schritt des ESGM besteht in einer qualitativen Bewertung von Geschäftsmodellen. Hierbei wird eine generische Nutzen-Aufwand-Analyse durchgeführt und abschlieBend werden die Bewertungen der einzelnen Geschäftsmodelle in einer Matrix visualisiert. Aus der Relation von Nutzen und Aufwand kann auf die Attraktivität im Sinne einer langfristigen Rentabilität einzelner Geschäftsmodelle geschlossen werden. Dies ermöglicht eine Vorauswahl für nähere quantitative Untersuchungen.

Die Grundlagen des qualitativen Bewertungsschemas des ESGM sind die Aufwand-Nutzen-Matrix von Knab et al. (2014) und die Scoring-Tabelle von Schallmo (2014a). AuBerdem wird das Schema mit der PESTEL-Methode erweitert. Der Scoring-Ansatz von Schallmo (2014a) wird dabei genutzt, um eine grobe Einschätzung und Bewertung der Parameter im Rahmen der Aufwand-Nutzen-Matrix von Knab et al. (2014) zu ermöglichen. Die PESTEL-Methode wird weiterhin genutzt, um Parameter wie den technischen Nutzen in die Bewertung einzubeziehen, und somit nicht ausschließlich kurzfristige ökonomische Parameter zu integrieren. Dies ermöglicht es, dass die durch ein Unternehmen geschaffenen ökonomischen, ökologischen und sozialen Werte berücksichtigt werden. Diese Aspekte der Nachhaltigkeit stellen zum einen ein Risiko bzw. Aufwand aber auch einen Nutzen dar. Die Berücksichtigung von wirtschaftlichen, sozialen, ökologischen und technologischen Aspekten bei der Analyse eines Geschäftsmodells gewinnt somit zunehmend an Bedeutung. Der geschaffene Kundennutzen eines Geschäftsmodells wird somit nicht mehr ausschließlich durch eine Komponente abgedeckt (Joyce und Paquin 2016). Zusätzlich wurden im Zuge eines durchgeführten Workshops mit Experten eines großem deutschen DAX Unternehmens noch die infrastrukturellen Hürden, als wichtige Bewertungsgrundlage identifiziert und in die Bewertungsmethode hinzugefügt.

Die Methode kombiniert so existierende qualitative Bewertungsverfahren, um eine Grobauswahl einzelner Geschäftsmodelle zu erreichen, die unter den sich ändernden Bedingungen der Energiewirtschaft attraktiv sind und langfristig Bestand haben können. Dies ist notwendig, da die 


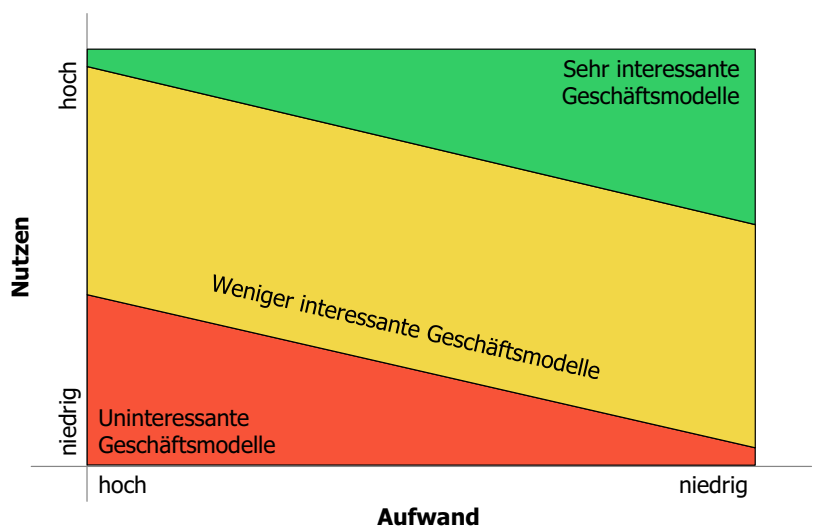

Abb. 1 Darstellung der Aufwand-Nutzen-Matrix zur qualitativen Bewertung von Geschäftsmodellen. (Quelle: Eigene Darstellung nach Knab et al. 2014)

kombinierten Verfahren einzeln nur eingeschränkt für die Energiewirtschaft nutzbar sind bzw. die aktuellen Trends nur unzureichend abgebildet werden können. Mit dieser Bewertungsmethode ist es möglich, Geschäftsmodelle anhand von wenigen Parametern qualitativ zu bewerten. Analog zu Knab et al. (2014) wird eine Matrix zur Einordnung von Geschäftsmodellen erstellt, die den Möglichkeitsraum in drei Bereiche unterteilt (Abb. 1). Die Fläche „Sehr interessante Geschäftsmodelle“, sowie die Fläche „Uninteressante Geschäftsmodelle" haben die gleiche Größe. Die übrige Fläche „Weniger interessante Geschäftsmodelle“ ist weitaus größer, da eine zu schnelle Klassifizierung der „Geschäftsmodelle mit hohem Potenzial“ oder „Geschäftsmodelle mit niedrigem Potenzial“ verhindert werden soll. Mit Hilfe dieser Matrix können Geschäftsmodelle nicht nur einzeln qualitativ im Vergleich zu bestehenden GM bewertet, sondern besonders mehrere neue Geschäftsmodelle miteinander verglichen werden.

Den Nutzen repräsentieren die in Tab. 1 aufgeführten Aspekte (Knab et al. 2014; Schallmo 2014b), wobei speziell die Aspekte Kostensenkungspotential, soziokultureller, technischer und Umweltnutzen auf den Kundennutzen insgesamt abzielen. Während ersterer direkt wirkt, können die weiteren sowohl direkt als auch indirekt (bspw. Verbesserung der Lebensqualität durch Umweltnutzen) wirken. Die verwendeten Nutzenaspekte zielen auf den betriebswirtschaftlichen Nutzen für das Unternehmen, das entsprechende Geschäftsmodelle realisiert bzw. erwägt, dies zu tun. Bei einigen Nutzenkategorien fließen hierbei volkswirtschaftliche Marktrahmenbedingungen ein. Die Bewertung zielt jedoch konsequent auf den Nutzen bzw. Mehrwert für das Unternehmen, der auch durch eine aus Unternehmenssicht positive Beeinflussung äußerer Einflussfaktoren gegeben sein kann. Beispielsweise kann ein Imagegewinn durch Generierung eines soziokulturellen Nutzens erreicht werden, welcher wiederum zur Kundenbindung oder Absatzsteigerung
Tab. 1 Nutzenaspekte der qualitativen Bewertung

\begin{tabular}{|c|c|}
\hline $\begin{array}{l}\text { Kostensenkungs- } \\
\text { potenzial }\end{array}$ & $\begin{array}{l}\text { Wie hoch sind die erwarteten Kostensenkungen des } \\
\text { Geschäftsmodells im Vergleich zu den anderen zu } \\
\text { bewertenden Geschäftsmodellen? }\end{array}$ \\
\hline Absatzpotenzial & $\begin{array}{l}\text { Wie groß ist das Absatzpotenzial des entstehenden } \\
\text { Produktes am Markt, in dem das Geschäftsmodell } \\
\text { Umsatz generieren kann? }\end{array}$ \\
\hline Imagepotenzial & $\begin{array}{l}\text { Wie hoch ist die Imageverbesserung eines Unter- } \\
\text { nehmens nach der Durchführung des Geschäftsmo- } \\
\text { dells? }\end{array}$ \\
\hline $\begin{array}{l}\text { Soziokultureller } \\
\text { Nutzen }\end{array}$ & $\begin{array}{l}\text { Wie hoch ist der soziokulturelle Nutzen für die } \\
\text { Gesellschaft durch die Umsetzung des Geschäfts- } \\
\text { modells? }\end{array}$ \\
\hline $\begin{array}{l}\text { Technischer } \\
\text { Nutzen }\end{array}$ & $\begin{array}{l}\text { Wie hoch ist der technische Nutzen durch das Ge- } \\
\text { schäftsmodell? } \\
\text { Generiert das Geschäftsmodell eventuell innovative } \\
\text { oder umweltfreundliche Produkte? } \\
\text { Bringt das Geschäftsmodell neue Werkstoffe oder } \\
\text { umweltfreundliche Energieträger hervor? } \\
\text { Wird mit der Realisierung des Geschäftsmodells } \\
\text { die Geschwindigkeit des Technologietransfers } \\
\text { beschleunigt? }\end{array}$ \\
\hline Umweltnutzen & $\begin{array}{l}\text { Wie hoch ist der Nutzen für die Umwelt? } \\
\text { Kann mit Hilfe des Geschäftsmodells ein Beitrag } \\
\text { zur Energiewende geleistet werden oder können } \\
\text { mit Hilfe des Geschäftsmodells Energieeinsparun- } \\
\text { gen getätigt werden? } \\
\text { Beeinflusst die Realisierung des Geschäftsmodells } \\
\text { in einer anderen Art und Weise sonst die Umwelt } \\
\text { positiv, wodurch eine höhere Akzeptanz zu erwar- } \\
\text { ten ist? }\end{array}$ \\
\hline
\end{tabular}

beitragen kann. Diese angewandte Bewertungssystematik zielt auf eine vergleichende Bewertung der Geschäftsmodelle ab. So werden die Geschäftsmodelle miteinander verglichen und entsprechend vergleichend bewertet.

Die Parameter bilden so den Nutzen des Geschäftsmodells umfassend $a b$, wobei regulatorische Treiber einzelne Nutzenaspekte verstärken können. Dies betrifft insbesondere die letzten drei Parameter, wodurch einzelne Win-WinSituationen entstehen können, in denen Geschäftsmodelle sowohl Kundennutzen generieren wie auch hinsichtlich der rechtlichen Vorgaben für ein Unternehmen förderlich sind.

Demgegenüber steht der in Tab. 2 abgebildete Aufwand (Knab et al. 2014; Schallmo 2014b).

Der Aufwand für ein Geschäftsmodell ergibt sich demnach aus verschiedenen Aspekten. So sind einerseits die Kosten für die Realisierung ein entscheidendes Kriterium. Weiterhin können technische Anforderungen über den Fähigkeiten eines Unternehmens liegen oder breit verteilt sein. Für die Bewertung eines Geschäftsmodells ist daher vorab abzuschätzen, ob die Anforderungen dem Stand der Technik entsprechen oder besondere Entwicklung erfordern, die einen besonderen Aufwand bedeuten (aber auch einen besonderen Nutzen generieren können). Im praktischen Anwendungsfall kann so ein Unternehmen direkt abschätzen, ob es das nötige Know-how besitzt. Analog ist die organisa- 
Tab. 2 Aufwandsaspekte der qualitativen Bewertung

\begin{tabular}{|c|c|}
\hline $\begin{array}{l}\text { Kosten der Umset- } \\
\text { zung }\end{array}$ & $\begin{array}{l}\text { Wie hoch sind die zusätzlichen Kosten im } \\
\text { Vergleich zwischen den zu bewertenden } \\
\text { Alternativen, welche die Realisierung des } \\
\text { Geschäftsmodells verursacht? }\end{array}$ \\
\hline $\begin{array}{l}\text { Technische Kom- } \\
\text { plexität }\end{array}$ & $\begin{array}{l}\text { Wie hoch ist die technische Komplexität für } \\
\text { die Realisierung des Geschäftsmodells? } \\
\text { Ist das benötigte Know-how vorhanden? }\end{array}$ \\
\hline $\begin{array}{l}\text { Organisatorische } \\
\text { Komplexität }\end{array}$ & $\begin{array}{l}\text { Wie hoch ist die organisatorische Komplexität } \\
\text { für die Realisierung, bzw. sind Prozessverän- } \\
\text { derungen notwendig? } \\
\text { Stellt die organisatorische Komplexität eine } \\
\text { zu hohe Markteintrittsbarriere für Unterneh- } \\
\text { men dar? }\end{array}$ \\
\hline $\begin{array}{l}\text { Rechtliche, politi- } \\
\text { sche Hürden }\end{array}$ & $\begin{array}{l}\text { Wie hoch sind die rechtlich, politischen Hür- } \\
\text { den, sind z. B. Gesetze, bzw. Subventionen } \\
\text { vorhanden, welche Substitut Produkte oder } \\
\text { Technologien fördern? }\end{array}$ \\
\hline $\begin{array}{l}\text { Soziokulturelle } \\
\text { Hürden }\end{array}$ & $\begin{array}{l}\text { Wie hoch sind die gesellschaftlichen Hürden, } \\
\text { ist z. B. eine Akzeptanz der Gesellschaft für } \\
\text { das Geschäftsmodell oder die Technologie } \\
\text { vorhanden? }\end{array}$ \\
\hline $\begin{array}{l}\text { Infrastrukturelle } \\
\text { Hürden }\end{array}$ & $\begin{array}{l}\text { Ist die notwendige Infrastruktur vorhanden, } \\
\text { bzw. sind nur kleine Anpassungen nötig oder } \\
\text { muss eine neue Infrastruktur implementiert } \\
\text { werden? }\end{array}$ \\
\hline
\end{tabular}

torische Komplexität zu bewerten. Weiterhin führen rechtliche oder soziokulturelle Hürden zu Aufwand hinsichtlich der Umsetzung eines Geschäftsmodells. Hierbei können einerseits vergleichende Bewertungen einen Aufschluss über die Stärke dieser Hürden geben, andererseits Abschätzungen durch rechtliche Analysen und Marktumfragen durchgeführt werden oder mit vorhandenen GM verglichen werden. Infrastrukturelle Hürden spielen gerade bei der Herstellung flüssiger und gasförmiger Energieträger eine bedeutende Rolle. Bei den infrastrukturellen Hürden, kann somit zum Beispiel der Aufwand für die Einspeisung in das Gasnetz vergleichend bewertet werden. Die Auswahl bzw. die Einteilung der Bewertungsparameter kann entsprechend des Anwendungsfalls angepasst werden. Die Ergebnisse der Bewertung lassen sich in der folgenden Matrix visualisieren. Es ist zu beachten, dass besonders Geschäftsmodelle im oberen rechten Bereich besonders interessant sind, da hier auch ein besonders hohes Potential vorliegt. Im oberen linken Bereich können Geschäftsmodelle liegen, die zwar interessant sind, deren Potential allerdings gering ist, solange keine Aufwandsreduktion ermöglicht wird. Geschäftsmodelle mit niedrigem Nutzen sind prinzipiell ungünstig, da hier ein niedriges Potential für die Geschäftsmodelle unterstellt werden muss.

\subsection{Quantitative Bewertung von Geschäftsmodellen}

Die quantitative Bewertung der Geschäftsmodelle durch das ESGM erfolgt anhand der DCF-Methode. Es werden zudem möglicherweise anfallende Investitionen, die mit der Auswahl eines Geschäftsmodells einhergehen berücksichtigt. Bei der Anwendung der DCF-Methode werden die den Geschäftsmodellen zugrunde liegenden Technologien, Produkte und Kosten berïcksichtigt. Zusätzlich werden Sensitivitätsanalysen integriert, um unsichere Entwicklungen bei der Planung neuer Geschäftsmodelle abbilden zu können.

Für das ESGM wurde ein Ansatz entwickelt, mit dem sich für verschiedene Erlös- und Kostenparameter automatisch Zahlungsreihen über einen Planungshorizont generieren lassen. Die grundlegende Systematisierung der Erlösund Kostenparameter ist in Tab. 3 dargestellt.

Generische Zahlungsströme, die unabhängig von der Preisentwicklung an verschiedenen energiewirtschaftlichen Märkten sind, wie z.B. Erlöse durch den Verkauf von Softwarelizenzen oder Kosten für Personal, können im Modell frei durch den Nutzer quantifiziert werden. Energiewirtschaftliche Zahlungsreihen hingegen, wie z.B. die Erlöse aus der Regelleistungsvermarktung, können über historische Markt- und technische Anlagendaten realistisch abgeschätzt werden.

Als Datengrundlage sind im Modell dafür:

- energieträgerspezifische Marktwerte und dynamische Preisspreads an der EPEX-Spot,

- Leistungs- und Arbeitspreise verschiedener Regelleistungsprodukte (Frequency Containment Reserves (FCR), automatic oder manual Frequency Restoration Reserves (aFRR, mFRR)),

- Daten zur Häufigkeit, -höhe und -dauer für Abrufe von Regelleistung und

- technologiespezifische Jahresvolllaststunden

hinterlegt. Weitere lassen sich problemlos ergänzen.

Grundsätzlich können Erlöse im Modell über drei generische Komponenten abgebildet werden. Dazu zählen Grundpreise, Arbeitspreise und Leistungspreise. Um zum Beispiel Erlöse aus einem Stromvertrag zu modellieren, könnte man neben einer monatlichen Grundgebühr einen Arbeitspreis

Tab. 3 Erlös- und Kostenparameter energiewirtschaftlicher Geschäftsmodelle (Ausschnitt)

\begin{tabular}{ll}
\hline Erlöse & Kosten \\
\hline $\begin{array}{l}\text { Grundgebühr (einmalig/ } \\
\text { wiederkehrend) }\end{array}$ & Variable Kosten (pro Anlage/ \\
Preis für elektrische Arbeit/ & Kunde) \\
Leistung & Einmalig pro Neukunde \\
Spotmarkthandel & \\
Regelleistung/Regelarbeit & Fixkosten \\
\hline
\end{tabular}


Tab. 4 Rentabilitätsmaße

\begin{tabular}{lll}
\hline Kapitalwert & $\begin{array}{l}\text { Gewinn, den ein Geschäftsmodell über die } \\
\mathrm{K}_{0} \text { in } €\end{array}$ & $K_{0}=I_{0}+\sum_{i=0}^{T} \frac{E_{t}-A_{t}}{(1+i)^{i}}$ \\
$\begin{array}{l}\text { Interner Zinssatz } \\
\text { IRR }\end{array}$ & $\begin{array}{l}\text { Rentabilität des durch die Investition gebun- } \\
\text { denen Kapitals }\end{array}$ & $K_{0}=I_{0}+\sum_{i=0}^{T} \frac{E_{t}-A_{t}}{(1+I R R)^{i}}=0$ \\
$\begin{array}{l}\text { Annuität } \\
\text { a in } €\end{array}$ & $\begin{array}{l}\text { Rechnerischer Gewinn pro Periode (ver- } \\
\text { teilt den Kapitalwert gleichmäßig auf den }\end{array}$ & $\begin{array}{l}a=K_{0} \cdot \frac{q^{t}(q-1)}{q^{t}-1} \\
\text { mit } q=1+i\end{array}$ \\
$\begin{array}{l}\text { Planungshorizont) } \\
\text { Amortisation }\end{array}$ & $\begin{array}{l}\text { Dauer, nach der sich die Anfangsinvestition } \\
\text { bezahlt macht }\end{array}$ & $-I_{0}+\sum_{t=1}^{T_{\mathrm{dyn}}} \frac{E_{t}-A_{t}}{(1+i)^{t}}>0$ \\
\hline
\end{tabular}

je abgesetzter Strommenge festlegen. Basierend auf der erwarteten jährlichen Kundenanzahl sowie den Wachstumsraten wird die Zahlungsreihe für den vorgegebenen Planungszeitraum generiert. Ähnlich könnte man vorgehen, um im Modell Erlöse aus dem Bereich Software-as-a-Service (SaaS) zu erfassen. Lizenzgebühren könnten beispielsweise als einmalige Grundgebühr gelten, Erlöse für die Wartung der Software als wiederkehrende Grundgebühr.

Für ausgewählte Produkte der Spot- und Regelleistungsmärkte sind im ESGM spezielle Berechnungslogiken implementiert. Basiert ein Geschäftsmodell beispielsweise auf Regelleistungserlösen wird zunächst die Leistung der für das Geschäftsmodell ausgewählten Technologien quantifiziert. Dabei werden technische Restriktionen sowie die von den Übertragungsnetzbetreibern vorgegebenen Mindestgebotsgrößen berücksichtigt. Erlöse für die Leistungsvorhaltung werden anschließend über historische Leistungspreise berechnet. Für die Regelleistungsprodukte aFRR und mFRR werden im Modell zusätzlich Erlöse aus der Leistungserbringung quantifiziert und jeweils nach der Regelrichtung differenziert. Dabei werden historische Arbeitspreise sowie typische Charakteristika der Abrufe berücksichtigt.

Kosten können über den entwickelten Ansatz produktspezifisch oder generisch modelliert werden. Bei der Berechnung produktspezifischer Kosten wird die Anzahl der Kunden bzw. der genutzten Technologieeinheiten als Bezugsgröße berücksichtigt. Abhängig von der gewählten Kostenart wird dann entweder die Neu- oder die Bestandskundenanzahl verwendet. Bei der Berechnung der allgemeinen Kosten wird im Modell zwischen Investitionskosten und Fixkosten differenziert.

Analoge Berechnungen sind auch für weitere Komponenten aus Tab. 3 möglich. Basierend auf den generierten Erlös- und Kostenreihen wird das Geschäftsmodell dann mithilfe der Rentabilitätsmaße aus Tab. 4 bewertet.

Das ESGM lässt die Eingabe von verschiedenen Geschäftsmodell-Konstellationen zu. Der Nutzer wird beim Entwurf neuer Geschäftsmodelle durch aufeinanderfolgende Abfrageformulare unterstützt, die sich interaktiv an seine Eingaben anpassen. Die Ergebnisse der Wirtschaftlichkeitsund Sensitivitätsanalyse werden im ESGM visualisiert (siehe Abb. 2).

\section{Demonstration des generischen Bewertungsmodells}

Das im vorherigen Kapitel vorgestellte ESGM soll mittels der Betrachtung von Geschäftsmodellen angewandt werden. Zu diesem Zweck wird auf Geschäftsmodelle aus den Bereichen Sektorenkopplung und digitale Plattformen der Energiewirtschaft zurückgegriffen. Beide Bereiche stehen beispielhaft für neuartige energiewirtschaftliche Geschäftsmodelle, welche durch die Energiewende begünstigt werden. Eine Übersicht zu den Komponenten der analysierten Geschäftsmodelle befindet sich im Anhang.

Im Fall der Sektorenkopplung werden Geschäftsmodelle aus dem Bereich Power-to-Gas (PtG) betrachtet. Das Fallbeispiel stellt einen Windparkbetreiber und dessen Möglichkeiten Wasserstoff zu erzeugen dar. Dabei bestehen fünf Optionen zur Ausgestaltung des Geschäftsmodells. Zunächst kann der erzeugte Wasserstoff im Rahmen des Geschäftsmodellprototyps Power-to-Gas direkt vermarktet werden (Wind-Wasserstoff). Im Rahmen von weiteren Umwandlungsschritten kann der Wassersoff weiter zu Methan (Wind-Methan) oder Powerfuels (Wind-Kraftstoff) veredelt werden. Zudem wird das Geschäftsmodell Power-to-Heat und damit der Einsatz in der Wärmebereitstellung (WindWärme) berücksichtigt. Letztlich wird auch die Option einer Weiterverarbeitung zu Ammoniak mitaufgenommen.

Im Fall der digitalen Geschäftsmodelle wird das Fallbeispiel eines plattformbasierten Geschäftsmodells betrachtet. Dabei werden zwei Ausgestaltungsformen der Plattform berücksichtigt. Der Basisfall besteht in dem Geschäftsmodellprototypen eines virtuellen Kraftwerks zum Erbringen von Systemdienstleistungen. Es sollen dezentrale Energieanalagen und Batteriespeicher zum Zweck der Regelenergiebereitstellung vernetzt werden. Ein weiteres betrachtetes Geschäftsmodell ist eine Community-Plattform aus der Gruppe Peer-to-Peer-Plattformen. Neben der Regelenergiebereitstellung können die an der Plattform teilnehmenden Anlageneigentümer ihren dezentral erzeugten Strom im Rahmen einer Community bilanziell teilen. 

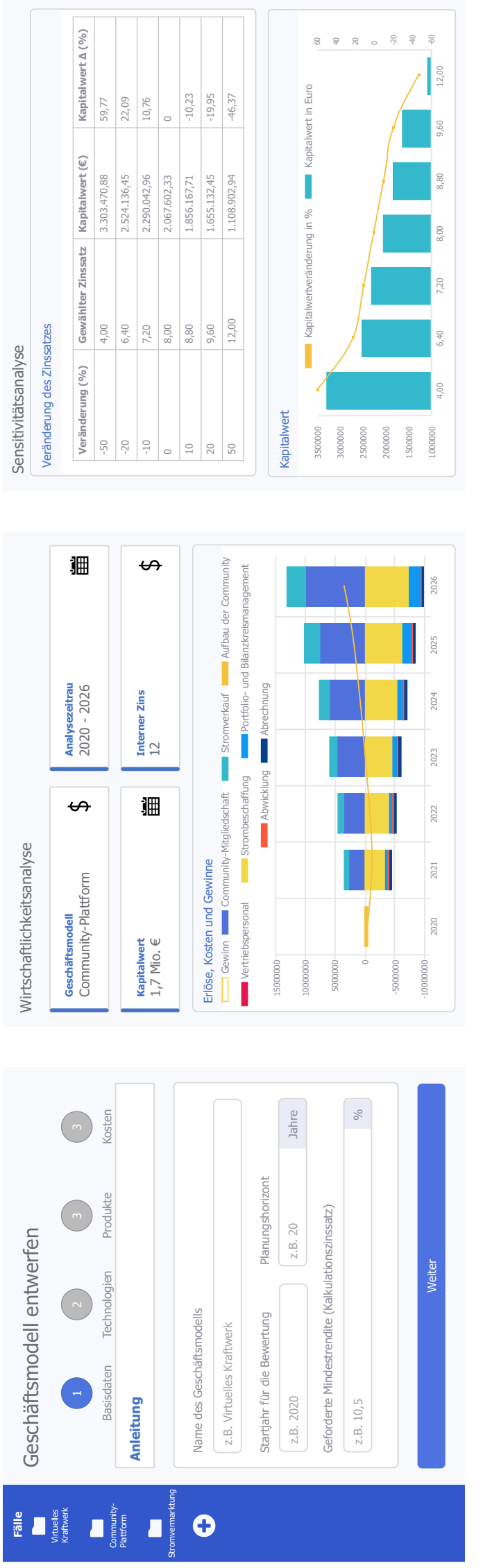

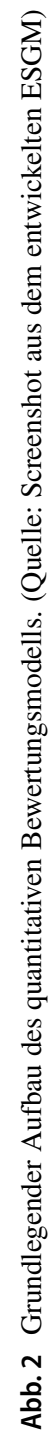




\subsection{Qualitative Analyseergebnisse}

Für die qualitative Bewertung der Attraktivität der Geschäftsmodelle wird der in Abschn. 3.1 beschriebene generische Ansatz genutzt. Dazu werden die genannten Geschäftsmodelle aus der betriebswirtschaftlichen Perspektive eines Unternehmens miteinander verglichen, dass erst in den Markt einsteigen will, allerdings Erfahrungen aus angrenzenden Märkten mitbringt (siehe oben). Nutzen und Aufwand der einzelnen GM werden entsprechend allgemein angenommen, können für spezialisierte Unternehmen allerdings deutlich abweichen. Im Folgenden ist dargestellt, wie die Attraktivität der einzelnen Geschäftsmodell bewertet wird. Hierbei werden einzelne Punkte detaillierter hervorgehoben.

Im Fall von PtG-Geschäftsmodellen sind deutliche Kostensenkungen bei der Elektrolyse zu erwarten (Milanzi et al. 2018). Zudem bieten die Geschäftsmodelle technischen und Umweltnutzen durch die die Möglichkeit, das Netz zu Zeiten hoher Erneuerbarer-Energien-Einspeisung entlasten zu können und grünen Wasserstoff bereitzustellen (Sternberg and Bardow 2015). Der Absatzmarkt für „grünen“ Wasserstoff ist derzeit noch nicht hinreichend vorhanden. Ein Marktwachstum wird für die kommenden Jahre angenommen. So ist eine Verbesserung des Mobilitätsverhaltens hin zu einer $\mathrm{CO}_{2}$-neutralen Mobilität der Gesellschaft vorstellbar (ENCON.Europe GmbH et al. 2018). Hinsichtlich soziokultureller Hürden des Geschäftsmodells ist ein enger Zusammenhang zum Image-Potential festzustellen. So ist eine mögliche Akzeptanz der Bevölkerung für Wind-Wasserstoff prinzipiell gegeben, allerdings kann ebenfalls eine zunehmende Ablehnung von Windparks in Deutschland festgestellt werden (Otto 2019).

Die rechtlichen Rahmenbedingungen stellen aktuell eine große Hürde für Umsetzungen von Wind-Wasserstoff-Anwendungen dar. Zwar ist der Elektrolyseur von Netzentgel- ten befreit, jedoch muss unter Umständen immer noch die EEG-Umlage gezahlt werden (Fraunhofer ISE 2019). Auch die Distribution des Wasserstoffes stellt aktuell noch eine große Hürde dar. Zwar existieren kleine regionale Wasserstoffnetze, jedoch sind keine überregionalen Netzstrukturen vorhanden. Es ist daher aktuell von Hürden im Hinblick auf die Infrastruktur für die Distribution auszugehen, die erst mittelfristig behoben werden könnten.

Zusammenfassend ist zu sagen, dass für das Geschäftsmodell ein relativ hoher Aufwand einem perspektivisch hohen Nutzen gegenübersteht. Das Geschäftsmodell ist daher prinzipiell attraktiv, allerdings stehen ihm zurzeit noch Hürden entgegen.

Für Geschäftsmodelle aus dem Bereichen Wind-Methan ist insgesamt von einem höheren Nutzen auszugehen, da u. a. ein etablierter Abnahmemarkt besteht. Die technische Komplexität liegt für das Geschäftsmodell zwar höher, allerdings ist eine Transportinfrastruktur bereits gegeben und kombinierte Prozesse (Elektrolyse und Methanisierung) bestehen bereits. Wind-Kraftstoff ist weiterhin mit einem im Vergleich noch höheren Aufwand zu bewerten, da hier ein weiterer Verfahrensschritt hinzukommt (bspw. FischerTropsch-Synthese). Auch wenn das Verfahren lange bekannt ist, sind aktuell nur wenige Anlagen realisiert und daher hohe Kosten für die Umsetzung anzunehmen. Beide Geschäftsmodelle fallen allerdings aufgrund des zu erwartenden höheren Nutzens (bestehende Abnahme) bereits in den Bereich der interessanten Geschäftsmodelle.

Im Vergleich zu den Geschäftsmodellen aus dem Bereich Wind-PtG lassen die Geschäftsmodelle virtuelles Kraftwerk und Community-Plattform einen deutlich geringeren Aufwand hinsichtlich der Realisierung erwarten. Trotzdem ist der Nutzen vergleichsweise groß und somit sind die Geschäftsmodelle überaus attraktiv. Der vergleichsweise geringe Aufwand ist in beiden Fällen primär mit einem geringen Kostenaufwand zu erklären, da zunächst kaum In-
Abb. 3 Ergebnisse der qualitativen Analyse. (Quelle: Eigene Darstellung nach Knab et al. 2014)

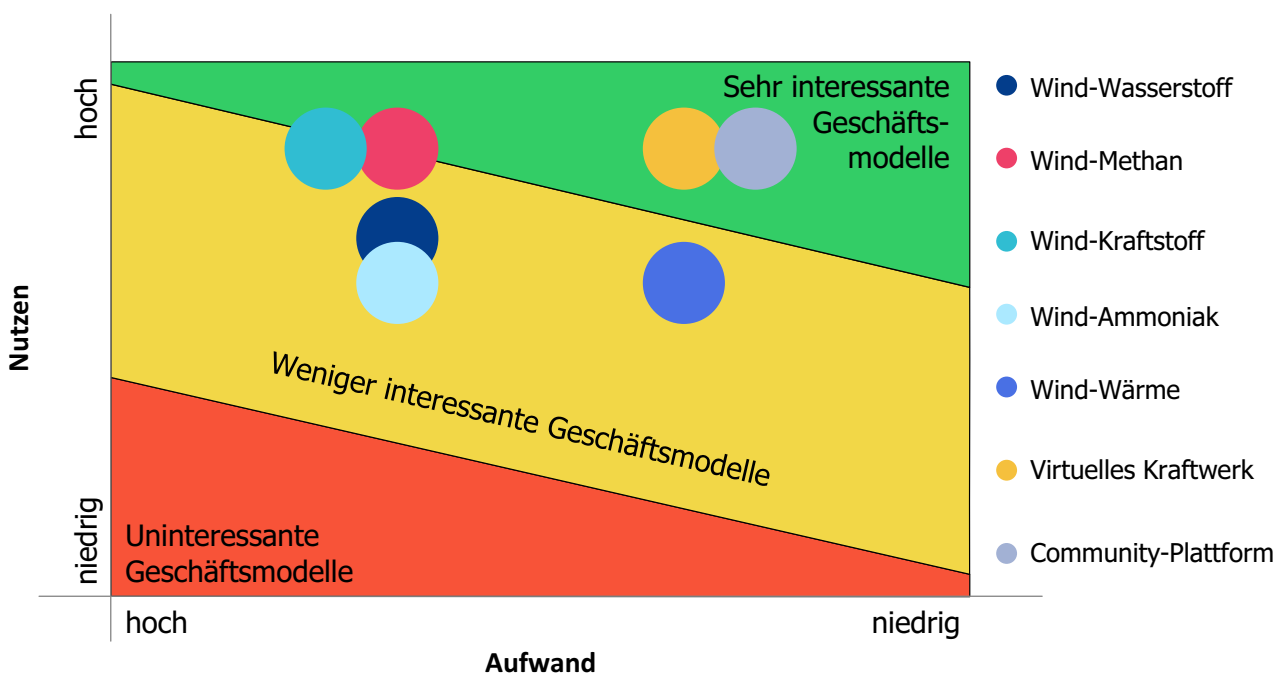


vestitionen getätigt werden müssen. Zudem ist in beiden Fällen eine einfache Skalierung auf mehrere Anlagen zu erwarten, die einen relativ hohen Nutzenzuwachs verspricht. Geschäftsmodelle aus dem Bereich Wind-Ammoniak und Wind-Wärme wurden im Vergleich zu Wind-Wasserstoff mit einem niedrigeren Nutzen bewertet. Dabei gleicht der Aufwand der Geschäftsmodelle aus dem Bereich WindAmmoniak denen aus dem Bereich Wind-Wasserstoff. Geschäftsmodelle aus dem Bereich Wind-Wärme wurden im Vergleich zu denen aus dem Bereich Wind-Wasserstoff mit einem niedrigeren Aufwand bewertet.

Abb. 3 fasst die Bewertung der Attraktivität in der vorgestellten Matrix zusammen. Es ist zu erkennen, dass besonders die Geschäftsmodelle Wind-Kraftstoff, Wind-Methan, virtuelles Kraftwerk und Community-Plattform einen hohen Nutzen versprechen und als besonders attraktiv eingestuft werden. Im Vergleich zum hohen Aufwand für WindKraftstoff und Wind-Methan weisen die Geschäftsmodelle virtuelles Kraftwerk und Community-Plattform ein noch besseres Nutzen-Aufwand-Verhältnis auf. Die beiden Geschäftsmodelle werden daher für die folgende quantitative Bewertung übernommen. ${ }^{1}$

\subsection{Quantitative Analyseergebnisse}

Das näher betrachtete Fallbeispiel des virtuellen Kraftwerks wird mithilfe des ESGMs weitergehend quantitativ analysiert. Dafür wird eine Nutzereingabe skizziert, die sich an Wirtschaftlichkeitsanalysen von virtuellen Kraftwerken (Reif und Fox 2014; Böttger et al. 2013; Loßner et al. 2015) sowie Herstellerangaben orientiert. Von Prognosen zur Entwicklung an den Regelleistungsmärkten wird abgesehen, da der Nutzer über die Eingabe unterschiedlicher Parameter flexibel verschiedene Szenarien im ESGM betrachten kann.

\subsubsection{Quantitative Analyse - Virtuelles Kraftwerk}

Die folgenden Eckdaten beschreiben das Geschäftsmodell. Das virtuelle Kraftwerk soll aus Solarfreiflächen, OnshoreWindparks, Biogasanlagen und Stromspeichern bestehen. Die Leistung der dezentralen Kundenanlagen wird aggregiert und gebündelt am Spot- und Regelleistungsmarkt vermarktet. Zu den angebotenen Produkten zählen u.a. FCR, aFRR und die Direktvermarktung. Insgesamt aggregiert das virtuelle Kraftwerk mehr als 50MW dezentrale Leistung. Der Betreiber des virtuellen Kraftwerks erwartet ein durchschnittliches Wachstum seines Anlagen-Portfolios um 5\% pro Jahr.

\footnotetext{
${ }^{1}$ Es ist zu beachten, dass diese Einschätzung auf Basis des aktuellen politischen Umfeldes und technologischen Stands getroffen wurde sowie auf Basis eines entscheidungsindifferenten Unternehmens.
}

Zusätzlich wird eine SaaS-Lösung für den Aufbau eigener virtueller Kraftwerke inklusive Softwarewartung für Stadtwerke und Energieversorgungsunternehmen angeboten. Im ersten Jahr wird mit zwei erfolgreichen Lizenzverkäufen, einem jährlichen Kundenwachstum von $50 \%$ und pro Lizenz mit einmaligen Einnahmen von $100.000 €$ gerechnet. Zusätzlich plant der Betreiber jährlich Erlöse durch die Softwarewartung in Höhe von 15\% der Lizenzgebühren zu erwirtschaften.

Für die Entwicklung der Software rechnet das Unternehmen mit einmaligen Investitionskosten von $700.000 €$ sowie 1 Mio. $€$ für die Anschaffung der notwendigen Räumlichkeiten, Rechnerhardware sowie der Präqualifikation des Regelleistungspools (Reif und Fox 2014 S. 40). Als fixe jährliche Wartungskosten für die Vernetzungs- und Kommunikationstechnik des virtuellen Kraftwerks veranschlagt der Betreiber $10 \%$ der Entwicklungskosten. Mit 30 Angestellten und einem durchschnittlichen Bruttolohn von $60.000 €$ rechnet das Unternehmen mit jährlichen Personalkosten von 1,8 Mio. $€$, die jährlich um $2 \%$ steigen. Die quantitative Analyse zeigt, dass das Geschäftsmodell bei einer Planungsdauer von sechs Jahren mit einem internen Zinssatz von $7 \%$ über der erwarteten Mindestrendite von $6 \%$ liegt. Die Anfangsinvestition von insgesamt 2,1 Mio. $€$ rentiert sich im letzten Jahr der Planungsdauer.

Ein detaillierter Blick auf die Zahlungsströme in Abb. 4 zeigt, dass die Wirtschaftlichkeit des Geschäftsmodells stark von den Einnahmen an den Regelleistungsmärkten abhängt. Die Auswertung verdeutlicht weiterhin, dass die a-Lösung einen großen Anteil des wirtschaftlichen Erfolgs des Geschäftsmodells ausmacht. Insbesondere die Erlöse aus den Wartungsverträgen für die Software gewinnen über die Planungsdauer zunehmend an Bedeutung, da das Bestandskundenportfolio wächst. Betrachtet man die Kostenkomponenten des Geschäftsmodells über die gesamte Planungsdauer wird deutlich, dass die Mess- und Steuerungstechnik gemeinsam mit der Strombeschaffung den größten Anteil der Kosten des virtuellen Kraftwerks ausmachen.

\subsubsection{Quantitative Analyse - Community-Plattform}

Im Rahmen des ergänzenden Geschäftsmodells Community-Plattform sollen die vernetzten Kunden als Gegenleistung für die Teilnahme an den Regelleistungsmärkten aus dem Strom des virtuellen Kraftwerks profitieren. Der Plattformbetreiber stellt dafür eine Community-Plattform zur Verfügung, über welche die Mitglieder ihren dezentral produzierten Strom bilanziell mit anderen Mitgliedern der Gemeinschaft teilen können. Die energiewirtschaftliche $\mathrm{Ab}$ rechnung, Strombeschaffung und das Bilanzkreismanagement erfolgt über den Plattformbetreiber. 
Erlöse, Kosten \& Gewinne in Euro

$\square$ Gewinn $\square$ FCR $\square$ aFRR Spotmarkthandel

Direktvermarktung VPP-SaaS Softwarewartung Investition

Softwareentwicklung Personalkosten@Strombeschaffung

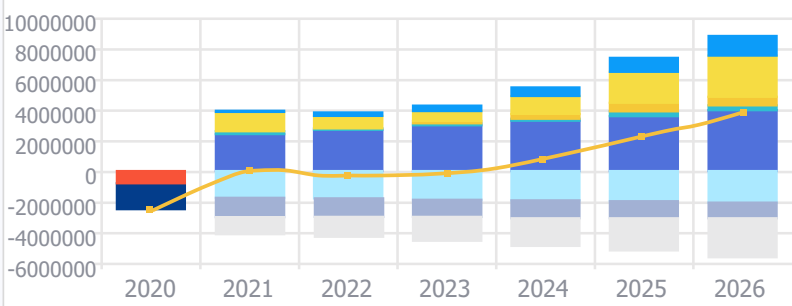

Cashflow in $€$

$\square$ Akkumulierter Cash Flow Cash Flow Diskontierter Cash Flow

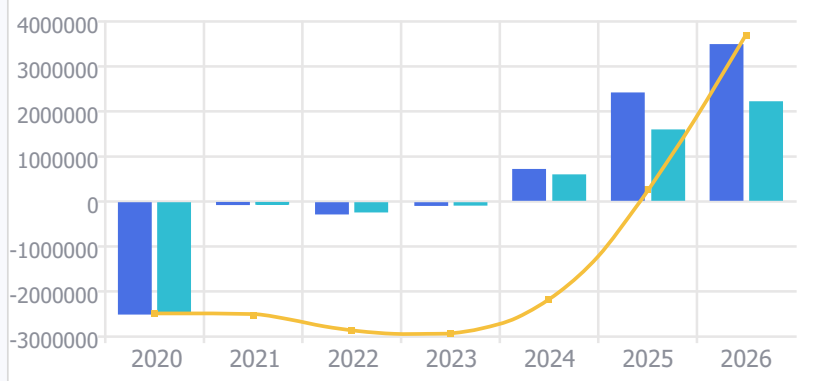

Einnahmenverteilung akkumuliert in $€$

FCR aFRR Spotmarkthandel Direktvermarktung VPP-SaaS Softwarewartung

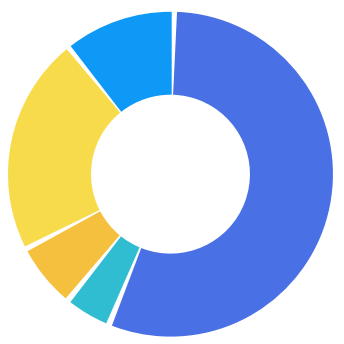

Kostenverteilung akkumuliert in $€$

- Investition Softwareentwicklung Personalkosten Strombeschaffung IT-Systeme@ Fernwirktechnik Betrieb Mess- und Kommunikationstechnik Erlösbeteiligung Anlageneigentümer

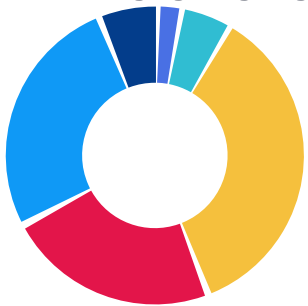

Abb. 4 Wirtschaftlichkeitsanalyse für das Virtuelle Kraftwerk. (Quelle: Screenshot aus dem entwickelten ESGM)

Erlöse, Kosten \& Gewinne in Euro

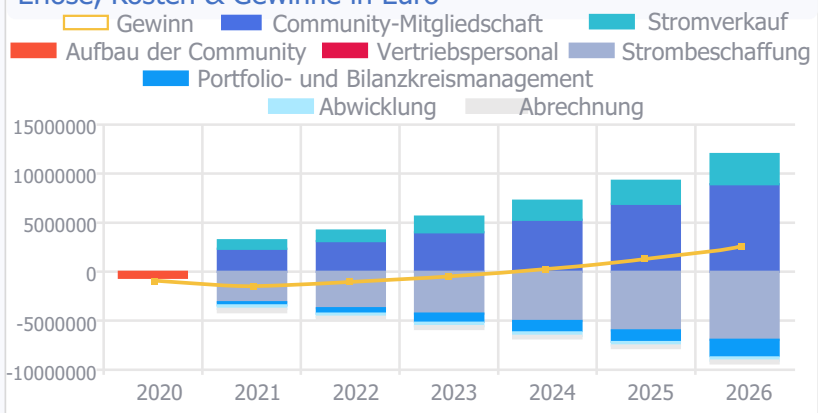

\section{Cashflow in $€$}

$\square$ Akkumulierter Cash Flow Cash Flow Diskontierter Cash Flow

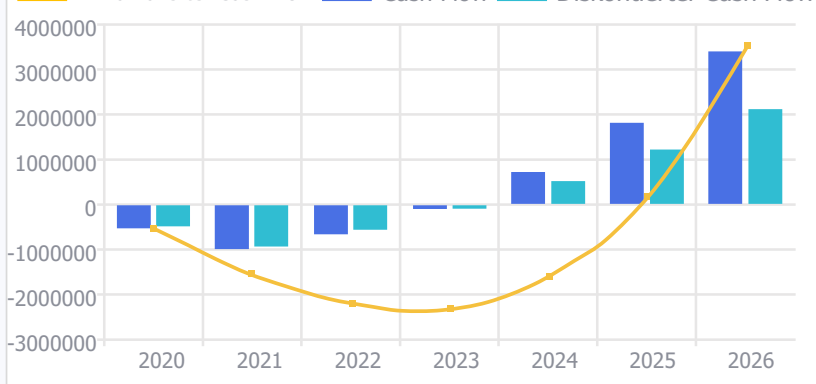

Einnahmenverteilung akkumuliert in $€$

Community-Mitgliedschaft Stromverkauf

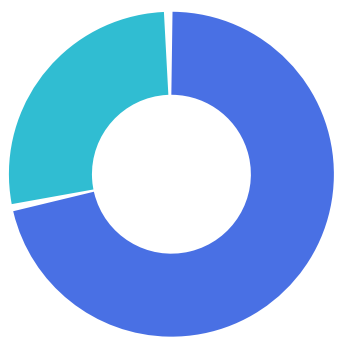

Kostenverteilung akkumuliert in $€$

Aufbau der Community Vertriebspersonal

Strombeschaffung — Portfolio- und Bilanzkreismanagement

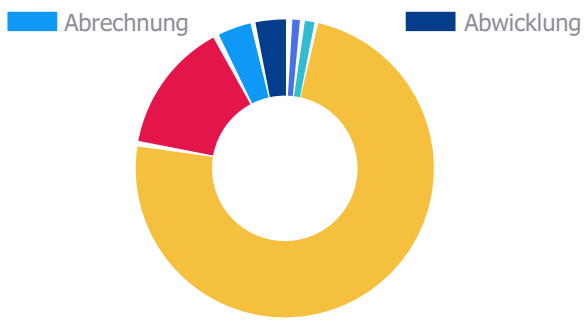

Abb. 5 Wirtschaftlichkeitsanalyse für die Community-Plattform. (Quelle: Screenshot aus dem entwickelten ESGM) 
Die Ergebnisse der quantitativen Analyse des Fallbeispiels Community-Plattform zeigen, dass das Geschäftsmodell mit $6 \%$ knapp über der geforderten Mindestrendite von $5 \%$ liegt und somit ebenfalls rentabel ist. Ein Blick auf die Zahlungsströme des Geschäftsmodells zeigt, dass die monatlichen Community-Gebühren über den Planungsverlauf die Kosten für die Strombeschaffung, die Abrechnung und das Bilanzkreismanagement decken (siehe Abb. 5). Über sinkende Kosten für die Verwaltung der Community kann der Plattformbetreiber ab der Hälfte des Planungszeitraumes positive Einnahmeüberschüsse erzielen. Die größten Kostentreiber sind die Strombeschaffung sowie das Portfolio- und Bilanzkreismanagement. Ausschlaggebend für die Rentabilität dieses Geschäftsmodells ist die Anzahl der über die Community vernetzten Kunden.

\section{Zusammenfassung und Ausblick}

In diesem Artikel wird ein methodischer Ansatz zur qualitativen und quantitativen Bewertung von Geschäftsmodellen der Energiewirtschaft aus betriebswirtschaftlicher Perspektive entwickelt. Ziel des Ansatzes ist es, eine schnelle und überschlägige Bewertung der Attraktivität und Erfolgsaussicht durchzuführen, um unternehmerische Entscheidungen vorzubereiten. Dieses Ziel wird, mit dem in diesem Artikel entwickelten ESGM, erreicht. In der qualitativen Bewertung kann eine erste Einschätzung generiert werden, ob das entwickelte Geschäftsmodell grundsätzlich für weitere Betrachtungen geeignet sein könnte. Aufgrund möglicher subjektiver Bewertungseinflüsse empfiehlt sich eine Bewertung im Vier- oder Mehr-Augen-Prinzip.

In einer quantitativen Bewertung kann das Geschäftsmodell auf seine wirtschaftliche Vorzugswürdigkeit untersucht werden. Hierbei werden mithilfe des DCF-Verfahrens insbesondere der Kapitalwert, die interne Verzinsung sowie die dynamische Amortisationsdauer des Vorhabens quantifiziert. Ferner werden relevante Kosten- und Erlösgrößen sowie Sensitivitäten der Eingangsparameter betrachtet und visualisiert. Das dahinter liegende ESGM ist generisch gehalten, sodass eine Vielzahl an unterschiedlichen Kostenbzw. Erlösparametern betrachtet werden können. Zudem ist der Grundaufbau des Tools auf Benutzerfreundlichkeit und eine ansprechende Visualisierung ausgerichtet.

Die Generalisierbarkeit des Tools wurde anhand von potenziellen Geschäftsmodellen der Sektorenkopplung und digitalen Plattformen der Energiewirtschaft demonstriert. Die Fallstudien für digitale Plattformlösungen werden in der qualitativen Analyse als interessante Geschäftsmodelle eingestuft. Die Geschäftsmodelle für Wind-Wasserstoff sind aufgrund des hohen Aufwands nicht für weitere quantitative Analysen herangezogen worden. Das virtuelle Kraftwerk und die Community-Plattform erweisen sich in der quantita- tiven Analyse als wirtschaftlich vorteilhaft mit einer jeweils positiven Rendite. Die Kosten für die Strombeschaffung sowie für die Mess- und Steuerungstechnik werden insgesamt als dominierende Kostenfaktoren identifiziert.

Weitere Anknüpfungsmöglichkeiten ergeben sich hinsichtlich der Hinterlegung weiterer Kosten- und Erlösmechanismen im Tool. Ferner könnten Prognosen zukünftiger Preisentwicklungen aus weiteren Modellen integriert und so verschiedene Zukunftsszenarien abgebildet werden. Nicht zuletzt könnten ergänzende Bewertungsmaßstäbe und Analyseansätze aus ökologischer oder sozialer Perspektive integriert werden, um zu einer möglichst ganzheitlichen Bewertung von Geschäftsmodellen zu kommen. Dies umfasst auch die Integration möglicher regulatorischer Rahmenbedingungen, die als Sensitivitäten im Tool abgebildet werden können.

Förderung Der vorliegende Artikel ist im Rahmen des durch das Bundesministerium für Bildung und Forschung (BMBF) geförderten Kopernikus-Projekts „Systemintegration“: Energiewende-Navigationssystem (ENavi) (Förderkennzeichen 03SFK4N0) entstanden. Die Autoren möchten sich für die Finanzierung der Arbeiten bedanken. 


\section{Anhang}

Übersicht über die mit dem ESGM analysierte Geschäftsmodelle der Energiewirtschaft. Zur Strukturierung ist auf das Business Model Framework for the Energy Transformation zurückgegriffen worden (Giehl et al. 2020).

Tab. 5 Ausprägungen der Komponenten des Geschäftsmodells Wind-Wasserstoff

\begin{tabular}{|c|c|c|c|c|c|}
\hline \multirow{2}{*}{\multicolumn{2}{|c|}{$\begin{array}{l}\text { Nutzenversprechen } \\
\text { Wasserstoff, Regelleistung, Netzstabilität, } \\
\text { Energiespeicher }\end{array}$}} & \multirow{2}{*}{\multicolumn{2}{|c|}{$\begin{array}{l}\text { Ertragsmodell } \\
\text { Energiepreis, Regelleistungs-/ } \\
\text { Regelenergiepreis }\end{array}$}} & \multirow{2}{*}{\multicolumn{2}{|c|}{$\begin{array}{l}\text { Kundensegment } \\
\text { Netzbetreiber, EVU, Händler, Energiever- } \\
\text { trieb, Autonutzer, Kraftstoffanbieter }\end{array}$}} \\
\hline & & & & & \\
\hline $\begin{array}{l}\text { Benötigte und offe- } \\
\text { rierte Daten }\end{array}$ & Einflussfaktoren & $\begin{array}{l}\text { Funktion im Wert- } \\
\text { schöpfungsnetzwerk }\end{array}$ & $\begin{array}{l}\text { Stufe im Wertschöp- } \\
\text { fungsnetzwerk }\end{array}$ & $\begin{array}{l}\text { Genutzte } \\
\text { Technologie }\end{array}$ & Partner \\
\hline $\begin{array}{l}\text { Regelleistungsdaten, } \\
\text { Preisdaten, Erzeu- } \\
\text { gungsdaten, Wetter- } \\
\text { daten }\end{array}$ & $\begin{array}{l}\text { Regulierung, Tech- } \\
\text { nologische Entwick- } \\
\text { lung }\end{array}$ & $\begin{array}{l}\text { Marktkoppler, Ser- } \\
\text { viceanbieter }\end{array}$ & $\begin{array}{l}\text { Erzeugung, Handel, } \\
\text { Speicher, Vertrieb }\end{array}$ & Elektrolyseur & $\begin{array}{l}\text { Anlagenbauer, Ver- } \\
\text { markter, Erzeuger, } \\
\text { Netzbetreiber, EVU, } \\
\text { Speicherbetreiber, } \\
\text { Börse }\end{array}$ \\
\hline
\end{tabular}

Tab. 6 Ausprägungen der Komponenten des Geschäftsmodells Wind-Methan

\begin{tabular}{|c|c|c|c|c|c|}
\hline \multirow{2}{*}{\multicolumn{2}{|c|}{$\begin{array}{l}\text { Nutzenversprechen } \\
\text { Synthetisches Methan, Regelleistung, Netz- } \\
\text { stabilität, Energiespeicher }\end{array}$}} & \multirow{2}{*}{\multicolumn{2}{|c|}{$\begin{array}{l}\text { Ertragsmodell } \\
\text { Energiepreis, Regelleistungs-/ } \\
\text { Regelenergiepreis }\end{array}$}} & \multirow{2}{*}{\multicolumn{2}{|c|}{$\begin{array}{l}\text { Kundensegment } \\
\text { Netzbetreiber, EVU, Händler, Energiever- } \\
\text { trieb }\end{array}$}} \\
\hline & & & & & \\
\hline $\begin{array}{l}\text { Benötigte und offe- } \\
\text { rierte Daten }\end{array}$ & Einflussfaktoren & $\begin{array}{l}\text { Funktion im Wert- } \\
\text { schöpfungsnetzwerk }\end{array}$ & $\begin{array}{l}\text { Stufe im Wertschöp- } \\
\text { fungsnetzwerk }\end{array}$ & $\begin{array}{l}\text { Genutzte } \\
\text { Technologie }\end{array}$ & Partner \\
\hline $\begin{array}{l}\text { Regelleistungsdaten, } \\
\text { Preisdaten, Erzeu- } \\
\text { gungsdaten, Wetter- } \\
\text { daten }\end{array}$ & $\begin{array}{l}\text { Regulierung, Tech- } \\
\text { nologische Entwick- } \\
\text { lung }\end{array}$ & $\begin{array}{l}\text { Marktkoppler, Ser- } \\
\text { viceanbieter }\end{array}$ & $\begin{array}{l}\text { Erzeugung, Handel, } \\
\text { Speicher, Vertrieb }\end{array}$ & $\begin{array}{l}\text { Elektrolyseur Me- } \\
\text { thanisisierunganlage }\end{array}$ & $\begin{array}{l}\text { Anlagenbauer, Ver- } \\
\text { markter, Erzeuger, } \\
\text { Netzbetreiber, EVU, } \\
\text { Speicherbetreiber, } \\
\text { Börse }\end{array}$ \\
\hline
\end{tabular}

Tab.7 Ausprägungen der Komponenten des Geschäftsmodells Wind-Kraftstoff

\begin{tabular}{|c|c|c|c|c|c|}
\hline \multirow{2}{*}{\multicolumn{2}{|c|}{$\begin{array}{l}\text { Nutzenversprechen } \\
\text { Synthetischer Kraftstoff, Regelleistung, } \\
\text { Netzstabilität, Energiespeicher }\end{array}$}} & \multirow{2}{*}{\multicolumn{2}{|c|}{$\begin{array}{l}\text { Ertragsmodell } \\
\text { Kraftstoffpreis, Regelleistungs-/ } \\
\text { Regelenergiepreis }\end{array}$}} & \multirow{2}{*}{\multicolumn{2}{|c|}{$\begin{array}{l}\text { Kundensegment } \\
\text { Netzbetreiber, EVU, Händler, Energiever- } \\
\text { trieb, Autonutzer, Kraftstoffanbieter }\end{array}$}} \\
\hline & & & & & \\
\hline $\begin{array}{l}\text { Benötigte und offe- } \\
\text { rierte Daten }\end{array}$ & Einflussfaktoren & $\begin{array}{l}\text { Funktion im Wert- } \\
\text { schöpfungsnetzwerk }\end{array}$ & $\begin{array}{l}\text { Stufe im Wertschöp- } \\
\text { fungsnetzwerk }\end{array}$ & $\begin{array}{l}\text { Genutzte } \\
\text { Technologie }\end{array}$ & Partner \\
\hline $\begin{array}{l}\text { Regelleistungsdaten, } \\
\text { Preisdaten, Erzeu- } \\
\text { gungsdaten, Wetter- } \\
\text { daten }\end{array}$ & $\begin{array}{l}\text { Regulierung, Tech- } \\
\text { nologische Entwick- } \\
\text { lung }\end{array}$ & $\begin{array}{l}\text { Marktkoppler, Ser- } \\
\text { viceanbieter }\end{array}$ & $\begin{array}{l}\text { Erzeugung, Handel, } \\
\text { Speicher, Vertrieb }\end{array}$ & $\begin{array}{l}\text { Elektrolyseur, Me- } \\
\text { thanisisierunganlage }\end{array}$ & $\begin{array}{l}\text { Anlagenbauer, Ver- } \\
\text { markter, Erzeuger, } \\
\text { Netzbetreiber }\end{array}$ \\
\hline
\end{tabular}

Tab. 8 Ausprägungen der Komponenten des Geschäftsmodells Wind-Ammoniak

\begin{tabular}{|c|c|c|c|c|c|}
\hline \multicolumn{2}{|l|}{ Nutzenversprechen } & \multicolumn{2}{|l|}{ Ertragsmodell } & \multicolumn{2}{|c|}{ Kundensegment } \\
\hline \multicolumn{2}{|c|}{$\begin{array}{l}\text { Chemische Grundstoffe, Regelleistung, Netz- } \\
\text { stabilität }\end{array}$} & \multicolumn{2}{|c|}{$\begin{array}{l}\text { Preis für Ammoniak, Regelleistungs-/ } \\
\text { Regelenergiepreis }\end{array}$} & \multicolumn{2}{|c|}{ Netzbetreiber, Händler, Chemieunternehmen } \\
\hline $\begin{array}{l}\text { Benötigte und offe- } \\
\text { rierte Daten }\end{array}$ & Einflussfaktoren & $\begin{array}{l}\text { Funktion im Wert- } \\
\text { schöpfungsnetzwerk }\end{array}$ & $\begin{array}{l}\text { Stufe im Wertschöp- } \\
\text { fungsnetzwerk }\end{array}$ & $\begin{array}{l}\text { Genutzte } \\
\text { Technologie }\end{array}$ & Partner \\
\hline $\begin{array}{l}\text { Regelleistungsdaten, } \\
\text { Preisdaten, Erzeu- } \\
\text { gungsdaten, Wetter- } \\
\text { daten }\end{array}$ & $\begin{array}{l}\text { Regulierung, Tech- } \\
\text { nologische Entwick- } \\
\text { lung }\end{array}$ & Marktkoppler & $\begin{array}{l}\text { Erzeugung, Handel, } \\
\text { Vertrieb angrenzen- } \\
\text { der Güter }\end{array}$ & Elektrolyseur & $\begin{array}{l}\text { Anlagenbauer, Er- } \\
\text { zeuger, Netzbetrei- } \\
\text { ber, EVU, Chemie- } \\
\text { unternehmen }\end{array}$ \\
\hline
\end{tabular}


Tab. 9 Ausprägungen der Komponenten des Geschäftsmodells Wind-Wärme

\begin{tabular}{|c|c|c|c|c|c|}
\hline \multirow{2}{*}{\multicolumn{2}{|c|}{$\begin{array}{l}\text { Nutzenversprechen } \\
\text { Erneuerbare Wärme, Regelleistung, NetzSta- } \\
\text { bilität, Flexibilität }\end{array}$}} & \multicolumn{2}{|l|}{ Ertragsmodell } & \multicolumn{2}{|l|}{ Kundensegment } \\
\hline & & \multicolumn{2}{|c|}{$\begin{array}{l}\text { Energiepreis, Regelleistungs-/ } \\
\text { Regelenergiepreis, Grundgebühr für Wär- } \\
\text { mebereitstellung }\end{array}$} & \multicolumn{2}{|c|}{$\begin{array}{l}\mathrm{HH}, \mathrm{GHD} \text {, Öffentliche Unternehmen, Indus- } \\
\text { trie, Städte und Kommunen, Netzbetreiber, } \\
\text { Energiespeicher }\end{array}$} \\
\hline $\begin{array}{l}\text { Benötigte und offe- } \\
\text { rierte Daten }\end{array}$ & Einflussfaktoren & $\begin{array}{l}\text { Funktion im Wert- } \\
\text { schöpfungsnetzwerk }\end{array}$ & $\begin{array}{l}\text { Stufe im Wertschöp- } \\
\text { fungsnetzwerk }\end{array}$ & $\begin{array}{l}\text { Genutzte } \\
\text { Technologie }\end{array}$ & Partner \\
\hline $\begin{array}{l}\text { Verbrauchsdaten, } \\
\text { Netzdaten, Preisda- } \\
\text { ten, Regelleistungs- } \\
\text { daten, Wetterdaten }\end{array}$ & Regulierung & $\begin{array}{l}\text { Marktkoppler, Er- } \\
\text { zeuger }\end{array}$ & $\begin{array}{l}\text { Handel, Speicher, } \\
\text { Erzeugung, Last- } \\
\text { management, Ver- } \\
\text { brauch }\end{array}$ & $\begin{array}{l}\text { Wärmepumpe, } \\
\text { Elektrodenheiz- } \\
\text { kessel }\end{array}$ & $\begin{array}{l}\text { Technologieanbieter, } \\
\text { Anlagenbetreiber, } \\
\text { Messdienstleister, } \\
\text { Netzbetreiber }\end{array}$ \\
\hline
\end{tabular}

Tab. 10 Ausprägungen der Komponenten des Geschäftsmodells virtuellen Kraftwerks-Systemdienstleistungen

\begin{tabular}{l} 
Nutzenversprechen \\
$\begin{array}{l}\text { Regelleistung, Vermarktung, Einnahmemög- } \\
\text { lichkeit, Netzstabilität, Demand Side Ma- }\end{array}$ \\
$\begin{array}{l}\text { nagement, Flexibilität, Direktbezug, Automa- } \\
\text { tisierung }\end{array}$ \\
$\begin{array}{l}\text { Benötigte und offerierte } \quad \text { Einfluss- } \\
\text { Daten }\end{array}$ faktoren \\
$\begin{array}{l}\text { Kapazitäts-, } \\
\text { Erzeugungs-, Netz- }\end{array}$ \\
$\begin{array}{l}\text { daten, Anlagendaten, } \\
\text { Preisdaten, Speicher- } \\
\text { daten, Verbrauchs-, }\end{array}$ \\
$\begin{array}{l}\text { Industrie-, Produktions-, } \\
\text { Regelleistungs-, Wetter- } \\
\text { daten, Nutzungsdaten }\end{array}$ \\
\hline
\end{tabular}

\section{Ertragsmodell Kundensegment}

Servicegebühren, Grundgebühr, Provision

HH, Industrie, GHD, Städte und Kommunen, Öffentliche Unternehmen, Netzbetreiber, Erzeuger, Speicherbetreiber

\section{Funktion im Wert- Stufe im Wertschöp- Genutzte Partner}

schöpfungsnetzwerk fungsnetzwerk Technologie

Marktmacher, Platt- Vertrieb, Handel, form, Serviceanbie- Verbrauch, Erzeuter gung, Speicher, Service, Lastmanagement
IoT-Plattform,

Mess- und Steue-

rungstechnik, Soft-

ware, Datenplatt-

form, Künstliche

Intelligenz
Netzbetreiber, Softwareanbieter, $\mathrm{Da}-$ tenplattform, Anlagenbetreiber, Messdienstleister, EVU, Börse, Händler

Tab. 11 Ausprägungen der Komponenten des Geschäftsmodells Community Plattform

\begin{tabular}{|c|c|c|c|c|c|}
\hline \multirow{2}{*}{\multicolumn{2}{|c|}{$\begin{array}{l}\text { Nutzenversprechen } \\
\text { Marktzugang, Lokales Energiesystem, direk- } \\
\text { ter Handel ohne Intermediär, Gemeinschaft, } \\
\text { Netzstabilität, Flexibilität, Transaktionsab- } \\
\text { wicklung, Kostenoptimierung }\end{array}$}} & \multirow{2}{*}{\multicolumn{2}{|c|}{$\begin{array}{l}\text { Ertragsmodell } \\
\text { Lizenzgebühren, Nutzungsgebühren, Ser- } \\
\text { vicegebühren, Grundgebühr, Provision }\end{array}$}} & \multicolumn{2}{|l|}{ Kundensegment } \\
\hline & & & & \multicolumn{2}{|c|}{$\begin{array}{l}\text { HH, Industrie, GHD, Städte und Kommunen, } \\
\text { Öffentliche Unternehmen, Prosumer, EVU, } \\
\text { Netzbetreiber, Versorger }\end{array}$} \\
\hline $\begin{array}{l}\text { Benötigte und offe- } \\
\text { rierte Daten }\end{array}$ & Einflussfaktoren & $\begin{array}{l}\text { Funktion im Wert- } \\
\text { schöpfungsnetzwerk }\end{array}$ & $\begin{array}{l}\text { Stufe im Wertschöp- } \\
\text { fungsnetzwerk }\end{array}$ & $\begin{array}{l}\text { Genutzte } \\
\text { Technologie }\end{array}$ & Partner \\
\hline $\begin{array}{l}\text { Kapazitäts-, Anla- } \\
\text { gen-Verfügbarkeits-, } \\
\text { Erzeugungs-, } \\
\text { Verbrauchs-, Preis- } \\
\text { daten, Anlagenda- } \\
\text { ten, Speicherdaten }\end{array}$ & $\begin{array}{l}\text { Regulierung, Tech- } \\
\text { nologische Entwick- } \\
\text { lung, Datenschutz }\end{array}$ & $\begin{array}{l}\text { Marktmacher, } \\
\text { Marktplattform, } \\
\text { Serviceanbieter, } \\
\text { Informations-Anbie- } \\
\text { ter/-verarbeiter }\end{array}$ & $\begin{array}{l}\text { Erzeugung, Ver- } \\
\text { brauch, Handel, } \\
\text { Plattform, Infor- } \\
\text { mationsanbieter, } \\
\text { Service, Messwesen }\end{array}$ & $\begin{array}{l}\text { IoT-Plattform, } \\
\text { Mess- und Steue- } \\
\text { rungstechnik, Soft- } \\
\text { ware, Smart Grid, } \\
\text { Datenplattform, } \\
\text { Blockchain, Künst- } \\
\text { liche Intelligenz }\end{array}$ & $\begin{array}{l}\text { Messstellenbetreiber, } \\
\text { Technologie-Soft- } \\
\text { wareanbieter, } \\
\text { Anlagenbetreiber, } \\
\text { Messdienstleister, } \\
\text { Netzbetreiber, EVU, } \\
\text { Datenplattform }\end{array}$ \\
\hline
\end{tabular}

\section{Literatur}

Agora Verkehrswende, Agora Energiewende, frontier Economics (2018) Die zukünftigen Kosten strombasierter synthetischer Brennstoffe, https://www.agora-energiewende.de/fileadmin2/ Projekte/2017/SynKost_2050/Agora_SynCost-Studie_WEB.pdf. Zugegriffen: 13. Aug. 2020

Anke J, Krenge J (2016) Prototyp eines Tools zur Abschätzung der Wirtschaftlichkeit von Smart Services für vernetzte Produkte. Tagungsband der Multikonferenz Wirtschaftsinformatik (MKWI) 2016, S. 1275-1286, Technische Universität Ilmenau, 09.-11. März 2016. Ilmenau

BDEW (2017) Blockchain in der Energiewirtschaft: Potenziale für Energieversorger. https://www.bdew.de/media/documents/BDEW_
Blockchain_Energiewirtschaft_10_2017.pdf. Zugegriffen: 15 . Febr. 2019

Böttger D, Hanemann P, Bruckner T (2013) Wirtschaftlichkeitsanalyse eines virtuellen Kraftwerks in Delitzsch innerhalb des EU-Projektes VIS NOVA. In: Werner A, Kühne S, Arnold G, Schmidt J (Hrsg) Energy EcoSystems Conference 2013 Leipzig. Leipziger Beiträge Zur Informatik, Bd. 42

Burger SP, Luke M (2017) Business model for distributed energy resources: a review and empirical analysis. Energy Policy 109:230-248. https://doi.org/10.1016/j.enpol.2017.07.007

DataPartner (2019) Invest for Excel—SoftwareTool von DataPartner. www.datapartner.fi/de/produkte/invest-for-excel. Zugegriffen: 2. Apr. 2020

Dena (2019) Blockchain in der integrierten Energiewende. https:// www.dena.de/fileadmin/dena/Publikationen/PDFs/2019/dena- 
Studie_Blockchain_Integrierte_Energiewende_DE4.pdf. Zugegriffen: 5. Jan. 2019

ENCON.Europe GmbH, IG BCE Innovationsforum Energiewende e. V., Mineralölwirtschaftsverband e. V., Mineralölwirtschaftsverband e. V. (2018) Potentialatlas für Wasserstoff, Analyse des Marktpotentials für Wasserstoff, der mit erneuerbaren Strom hergestellt wird, im Raffineriesektor und im zukünftigen Mobilitätssektor. https://www.innovationsforum-energiewende.de/ fileadmin/user_upload/Potentialstudie-fuer-gruenen-Wasserstoffin-Raffinerien.pdf. Zugegriffen: 13. Aug. 2020

Giehl J, Göcke H, Grosse B, Kochems J, Müller-Kirchenbauer J (2020) Survey and classification of business models for the energy transformation. Energies 13:2981. https://doi.org/10.3390/ en13112981

Jentsch M, Trost T (2014) Analyse von Power-to-Gas-Energiespeichern im regenerativen Energiesystem. im Auftrag des Bundesministerium für Wirtschaft und Energie (BMWi), Fraunhofer-Institut für Windenergie und Energiesystemtechnik (IWES), Kassel, Förderkennzeichen 0325275B

Joyce A, Paquin RL (2016) The triple layered business model canvas: a tool to design more sustainable business models. J Clean Prod 135:1474-1486. https://doi.org/10.1016/j.jclepro.2016.06.067

Khatib H (2014) Economic evaluation of projects in the electricity supply industry, 3. Aufl. IET power and energy series. The Institution of Engineering and Technology, London, United Kingdom

Knab S, Rohrbeck R, Konnertz L (2014) Kooperative Geschäftsmodellentwicklung für systemische Nachhaltigkeitsinnovationen: Eine Fallstudie im deutschen Smart Energy-Markt. In: Schallmo D (Hrsg) Kompendium Geschäftsmodell-Innovation: Grundlagen, aktuelle Ansätze und Fallbeispiele zur erfolgreichen Geschäftsmodell-Innovation. Springer Gabler, Wiesbaden, S $283-317$

Konstantin P (2009) Praxisbuch Energiewirtschaft: Energieumwandlung, -transport und -beschaffung im liberalisierten Markt, 2. Aufl. Springer, Berlin, Heidelberg

Kost C, Schick C, Grosse B, Kochems J (2019) Abschlussbericht zum ENavi-Schwerpunkt Wärmewende, Sektorkopplung, Nutzerintegration \& flexible, intelligente Steuerung. Fraunhofer ISE, Freiburg https://doi.org/10.24406/ise-n-574616

Liu J, Zhang N, Kang C, Kirschen D, Xia Q (2017) Cloud energy storage for residential and small commercial consumers: a business case study. Appl Energy 188:226-236. https://doi.org/10.1016/j. apenergy.2016.11.120

Löbbe S, Hackbarth A (2017) Geschäftsmodelle in der Energiewirtschaft: Ein Kompendium von der Methodik bis zur Anwendung. Reutlinger Diskussionsbeiträge zu Marketing \& Management, Bd. 2017:3. https://doi.org/10.15496/publikation-17713

Loßner M, Böttger D, Bruckner T (2015) Wirtschaftliches Potential virtueller Kraftwerke im zukünftigen Energiemarkt - Eine szenariobasierte und modellgestützte Analyse. ZfE 39:115-132. https:// doi.org/10.1007/s12398-015-0153-4

Margaria-Steffen T, Boßelmann S, Wickert A (2015) Der Business Model Developer - Entwicklung eines Tools zur Erstellung und
Analyse von Geschäftsmodellen. In: Eppinger E, Halecker B, Hölzle K, Kamprath M (Hrsg) Dienstleistungspotenziale und Geschäftsmodelle in der Personalisierten Medizin. Springer, Wiesbaden, S 95-115 https://doi.org/10.1007/978-3-658-08403$5 \_4$

Michaelis J, Junker J, Wietschel M (2013) Eine Bewertung der Regelenergievermarktung im Power-to-Gas-Konzept. ZfE 37:161-175. https://doi.org/10.1007/s12398-013-0113-9

Milanzi S, Spiller C, Grosse B, Hermann L, Müller-Kirchenbauer J (2018) Technischer Stand und Flexibilität des Power-to-Gas-Verfahrens https://doi.org/10.5281/ZENODO.2620254

Noack C, Burggraf F, Hossein S, Lettenmeier P, Kolb S, Belz S, et al (2015) Studie über die Planung einer Demonstrationsanlage zur Wasserstoff-Kraftstoffgewinnung durch Elektrolyse mit Zwischenspeicherung in Salzkavernen unter Druck. Projektbericht im Auftrag des Bundesministerium für Wirtschaft und Energie (BMWi). DLR, Stuttgart.

Osterwalder A, Pigneur Y (2013) Business model generation A handbook for visionaries, game changers, and challengers. John Wiley \& Sons, New York, NY

Otto A (2019) Landschaft und der Ausbau der Windenergie. In: Kühne O, Weber F, Berr K, Jenal C (Hrsg) Handbuch Landschaft. RaumFragen: Stadt - Region - Landschaft. Springer VS, Wiesbaden

Porter ME (2008) The five competitive forces that shape strategy. Harv Bus Rev 86(1):78-93

PWC (2018) eValuation Startup-Identifying value. Creating opportunities. https://startup.pwc-evaluation.com/de. Zugegriffen: 2. Apr. 2020

Quaschning V (2015) Regenerative Energiesysteme: Technologie Berechnung - Simulation, 9. Aufl. Hanser, München

Reif B, Fox A (2014) Eine Analyse zur Wirtschaftlichkeit von virtuellen Kraftwerken. Schriften zur Finanzwirtschaft. Technische Universität Ilmenau, Ilmenau

Schallmo D (2014a) Theoretische Grundlagen der GeschäftsmodellInnovation - Definitionen, Ansätze, Beschreibungsraster und Leitfragen. In: Schallmo D (Hrsg) Kompendium Geschäftsmodell-Innovation: Grundlagen, aktuelle Ansätze und Fallbeispiele zur erfolgreichen Geschäftsmodell-Innovation. Springer Gabler, Wiesbaden, S 1-30

Schallmo D (2014b) Checklisten und Erläuterungen zum Kompendium Geschäftsmodell-Innovation. In: Schallmo D (Hrsg) Kompendium Geschäftsmodell-Innovation: Grundlagen, aktuelle Ansätze und Fallbeispiele zur erfolgreichen Geschäftsmodell-Innovation. Springer Gabler, Wiesbaden, S 441-450

Sternberg A, Bardow A (2015) Power-to-what?-environmental assessment of energy storage systems. Energy Environ Sci 8:389-400. https://doi.org/10.1039/C4EE03051F

Zeller M (2014) Analyse und Simulation von Geschäftsmodellen für Elektrizitätsvertriebsunternehmen: Untersuchungen für die Implementierung von Smart Metern. Univ.-Verl. der TU Berlin, Berlin 